\title{
Optimization performance of plain journal bearings with partial wall slip
}

Shuhui Cui ${ }^{\mathrm{a}, \mathrm{b}}$, Chuanwei Zhang ${ }^{\mathrm{a} *}$, Michel Fillon ${ }^{\mathrm{b}}$, and Le Gu${ }^{\mathrm{a}}$

${ }^{a}$ MIIT Key Laboratory of Aerospace Bearing Technology and Equipment, Harbin Institute of Technology, Harbin 150001, China

b Pprime Institut, CNRS-Université de Poitiers-ISAE-ENSMA, Futuroscope Chasseneuil, 86962, France

*Corresponding author: zhchwei@hit.edu.cn

\begin{abstract}
In present work, in order to optimize partial slip zone for heavily loaded journal bearings, an isothermal hydrodynamic model considering wall slip occurring at oil-bush interface is built. Two-component slip model is utilized to calculate slip velocity. The performances of the journal bearing in different slip zone shapes and eccentricity ratios are evaluated. The optimal slip parameters in a range of eccentricity ratios are selected by considering their effects on hydrodynamic behavior, such as hydrodynamic pressure, load carrying capacity and friction coefficient/torque. In addition, the application of optimal partial slip zone is presented. Results show that the optimal range of slip zone in high eccentricity ratio is quite restricted. The slip zone should be located at the convergent region of the film. The circumferential end of slip zone should be close to the position of minimum film thickness. It is better to widen the axial length of slip zone, i.e. rectangular shape could better improve bearing performance.
\end{abstract}

Keywords: heavily loaded journal bearing, partial wall slip, mixed lubrication, simulation model

\section{Introduction}

In hydrodynamic bearings, coatings have been widely used due to their helpfulness to improve the tribological performance and prolong the serving life of bearings. Many kinds of plastic coatings, such as polytetrafluoroethylene (PTFE) and polyetheretherketone (PEEK), have lower surface energy and non-stick property, which are very different from the metal ones. For heavily loaded hydrodynamic journal bearings under high speed, wall slip is easier to occur, and the traditional nonslip boundary condition is not appropriate in the condition of high-speed sliding bearing with small gap [1]. Therefore, the influence of wall slip at fluid-solid interface is necessary to be considered.

In many literatures, experimental studies have been conducted to investigate the occurrence and influence of wall slip at the fluid-coating interface. Yu et al.[2] developed a rheometer and tested the perfluoropolyethers (PFPE) coated sample with the slip length in the order of $10 \mu \mathrm{m}$. They indicated that the critical shear rate of water was higher than that of PC and 3\# mineral oil. Jin et al.[3] tested that the critical shear stress at the oil-PTFE interface of a plastic journal bearing was about 1126.94 Pa. It was proved that, in hydrodynamic lubrication, the wall slip could decrease friction coefficient [4], but this reduction became smaller in mixed lubrication [5]. Ryan et al. [6] studied the Stribeck curve of the coated/uncoated surface and steel with a ball-on-flat test. They observed that when the film thickness was small, the micro-texture and coatings would affect the traction coefficient. With the lubricant of PAO oil, the $\mathrm{Cr}_{\mathrm{x}} \mathrm{N}$ coatings could decrease the traction coefficient more than DLC coatings.

Some slip models have been applied in the studies of hydrodynamic bearings. The modified Reynolds equation is deduced based on the Navier-Stokes equation [7-8], critical shear stress slip model [9-12] and two-component slip model [1315]. Cheng et al. [16] presented a slip model between cavitation air and lubricant oil to solve the problem of slip occurring in the cavitation zone. They deduced the Reynolds equation from Navier-Stokes equation considering both cavitation and wall slip. Jao et al. [17] studied the effect of anisotropy slip combined with roughness on the lubrication behavior of journal bearings. They studied the coefficients of the average Reynolds equation and concluded that the wall slip could offset part effect of roughness. Moreover, it was found that wall slip was easier to occur in the zones owning high shear stress gradient 
and small film thickness. The special oil wedge and high eccentricity ratio could decrease the influence of wall slip. In addition, the rupture zone and the maximum pressure are also affected by wall slip [11].

For journal bearings, the optimal designs of wall slip parameters have already been investigated, because the performance of the slip zone is significantly affected by working conditions. Ma et al. [18] systematically investigated and obtained the optimal parameters of the slip zone for the eccentricity ratios from 0 to 0.8 . They also indicated that if the wall slip occurred on the oil-shaft interface, there were almost no extra bearing load. Zhang et al. [15] studied the effects of water-bush boundary slip on the high-speed water lubricated journal bearings under low eccentricity ratios. They found it would decrease the load carrying capacity for full slip configuration on the bush, but an appropriate partial slip zone could improve the performance of the bearing. Rao [19] analyzed the effect of partial slip immediately followed by a single grooved on the journal bearing and found that the maximum hydrodynamic pressure was larger in partial slip condition. However, the shear stress was large before the slip/nonslip interface and obviously decreased in the nonslip zone. Fortier et al. [20] evaluated the performance of the load carrying capacity, friction force, and the leakage rate. They concluded that for low and moderate eccentricity ratios, slip boundary condition had an advantage for the improvement of load carrying capacity and friction coefficient. However, for the eccentricity ratios larger than 0.92, the effect of slip became much smaller. Lv et al.[12] demonstrated that, in mixed lubrication regime, wall slip occurring at thin polymer film coated on metallic bush could decrease the load bearing capacity and increase the friction coefficient. Wang et al. [21] found that with an increasing eccentricity ratio, the slip was more difficult to occur, leading to an increase in load capacity in a parallel sliding gap.

In the current work, further optimization of the partial wall slip zone for high eccentricity ratios is studied by considering the surface roughness. The average Reynolds equation considering wall slip is applied and solved by finite element method (FEM). The optimal slip zone parameters for moderate shaft eccentricity ratios are first obtained. Then, the optimal parameters are imposed on the working conditions at high eccentricity ratios. Thirdly, the optimal slip zone parameters for high eccentricity ratio are chosen to investigate the performance in moderate eccentricity ratio. Finally, the best slip zone parameters for a range of eccentricity ratios are determined.

\section{Model and formulation}

\subsection{Geometry model}

In order to shape the slip zone at the coordinate of $\theta$, we defined three coefficients of $\alpha=B_{\mathrm{s}} / B_{1}(0 \leq \alpha \leq 2), \lambda=L_{1} / L_{\mathrm{s} 1}(0 \leq \lambda)$, and $\gamma=L_{\mathrm{s} 1} / L(0 \leq \gamma \leq 1)$ as shown in Fig.1. $\alpha<1$ and $\alpha>1$ indicate that the slip zone locates at the convergent region and divergent region of the film respectively. $\lambda>1$ means the end width $L_{1}$ is larger than the front width $L_{\mathrm{s} 1} . B_{1}$ is the length from attitude angle $\varphi$ to the minimum film thickness position. $L$ is bearing width, $\omega$ is shaft velocity, $e$ is eccentric distance and $R$ is shaft radius.
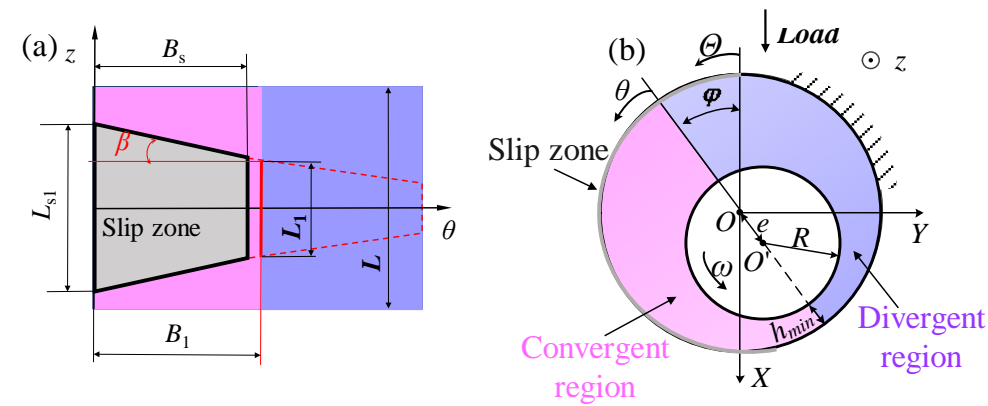

Fig. 1. The slip zone at the oil-bush interface

\subsection{Governing equation}

The lubricant is assumed to be incompressible and Newtonian and, the flow regime is laminar. The modified Reynolds 
equation considering both slip and surface roughness [23] is used as follows

$$
\frac{\partial}{\partial x}\left(\Phi_{\theta} \frac{h^{3}}{\eta} \frac{\partial p}{\partial x}\right)+\frac{\partial}{\partial z}\left(\Phi_{z} \frac{h^{3}}{\eta} \frac{\partial p}{\partial z}\right)=6 \Phi_{c}\left(u_{0}+u_{s}\right) \frac{\partial h}{\partial x}+\sigma \frac{\partial\left[\Phi_{s}\left(u_{0}+u_{s}\right)\right]}{\partial x}+6 \Phi_{c} h\left(\frac{\partial u_{s}}{\partial x}+\frac{\partial w_{s}}{\partial z}\right)+\xi p^{-}
$$

where $p$ is hydrodynamic pressure. $h$ is film thickness. $\eta$ is the dynamic viscosity of lubricant. $u_{0}$ is the velocity of shaft surface. $u_{s}$ and $w_{s}$ are the slip velocities in circumferential and axial directions. $\xi P^{-}$is the penalty item which could make flow mass conservation [24]. $\xi$ is an infinitely positive number and $p^{-}$represents the negative pressure in cavitation zone. $\Phi_{c}$ is the contact coefficient, $\Phi_{\theta}, \Phi_{z}$, and $\Phi_{s}$ are the flow rate coefficients that can be determined in Ref. [23] and [26]. $\sigma$ is the standard deviation of roughness asperity height, which is set to be $1 \mu \mathrm{m}$.

Taking the dimensionless parameters as $\bar{Z}=z / L, H=h / C, \psi=C / R, P=p /\left(\eta \omega / \psi^{2}\right), U=u_{0} /(R \omega), U_{s}=u_{s} /(R \omega)$, and $W_{s}=w_{s} /(R \omega)$, where $C$ is the radial clearance of the bearing, the dimensionless average Reynolds equation is obtained as

$$
\frac{\partial}{\partial \theta}\left(\Phi_{\theta} H^{3} \frac{\partial P}{\partial \theta}\right)+\left(\frac{R}{L}\right)^{2} \frac{\partial}{\partial \bar{Z}}\left(\Phi_{z} H^{3} \frac{\partial P}{\partial \bar{Z}}\right)=6 \Phi_{c}\left(U+U_{s}\right) \frac{\partial H}{\partial \theta}+\frac{\sigma}{C} \frac{\partial\left[\Phi_{s}\left(U_{0}+U_{s}\right)\right]}{\partial \theta}+6 \Phi_{c} H\left(\frac{\partial U_{s}}{\partial \theta}+\frac{R}{L} \frac{\partial W_{s}}{\partial \bar{Z}}\right)+\xi P^{-}
$$

In order to obtain the wall slip velocity, two-component slip model [13] is used. The shear stress is expressed by

$$
\tau_{c}=\tau_{c o}+\frac{\eta}{b} u_{s}
$$

where $\tau_{c o}$ is the critical shear stress at which wall slip occurs. $b$ is slip length. Wall slip begins when the fluid-solid shear stress is larger than the critical shear stress of $\tau_{c o}$. The slip velocity $u_{s}$ increases linearly with the increasing shear stress at a constant slip length of $b$.

The wall slip velocity in circumferential direction is

$$
u_{s}=\frac{u_{0}-\frac{1}{\eta} \frac{\partial p}{\partial x} \frac{h^{2}}{2}-\operatorname{sgn}\left(u_{s}\right) \frac{h \tau_{c o}}{\eta}}{(1+h / b)}
$$

where $\operatorname{sgn}\left(u_{s}\right)$ is the $\operatorname{sign}$ function for $\operatorname{sgn}\left(u_{s}>0\right)=1, \operatorname{sgn}\left(u_{s}<0\right)=-1$ and $\operatorname{sgn}\left(u_{s}=0\right)=0$.

As shown in Eq.(4), slip velocity is directly influenced by the gradient of hydrodynamic pressure $\mathrm{d} p / \mathrm{d} x$, the film thickness $h$, the critical shear stress $\tau_{c o}$, and the slip length $b$, which are discussed in section 3.

The dimensionless parameters of shear stress and slip length are

$$
\tau=\tau_{0} \bar{\tau}=\frac{\eta R \omega}{C} \bar{\tau}, \quad b=C \bar{b}
$$

The dimensionless wall slip velocity in circumferential direction is related to the HD pressure gradient

$$
\begin{aligned}
& U_{s}=\frac{U_{0}-\frac{\partial P}{\partial \theta} \frac{H^{2}}{2}-H \bar{\tau}_{c o}}{(1+H / \bar{b})}, \quad \text { if } u_{s}>0 \text { and } \frac{\partial P}{\partial \theta}<\frac{2}{H^{2}}\left(U_{0}-H \bar{\tau}_{c o}\right), \\
& U_{s}=\frac{U_{0}-\frac{\partial P}{\partial \theta} \frac{H^{2}}{2}+H \bar{\tau}_{c o}}{(1+H / \bar{b})}, \quad \text { if } u_{s}<0 \text { and } \frac{\partial P}{\partial \theta}>\frac{2}{H^{2}}\left(U_{0}+H \bar{\tau}_{c o}\right), \\
& U_{s}=0, \quad \text { if } u_{s}=0 \text { and } \frac{\partial P}{\partial \theta}=\frac{2}{H^{2}} U_{0}
\end{aligned},
$$

Similarly, the slip velocity $w_{s}$ in axial direction can be expressed in the same way.

\subsection{Solution procedure}

The modified Reynolds equation of Eq.(2) is solved by finite element method (FEM) with a four-node element. The detailed solution is presented in Ref [25].

The corresponding matrix equation for the four-node element is 


$$
\left[K_{4 \times 4}\right]\left\{\delta P_{4 \times 1}\right\}=R_{4 \times 1}
$$

The pressure is expressed by $P=P_{0}+\delta P$ and $\nabla P=\nabla P_{0}+\nabla \delta P . P_{0}$ and $\delta P$ are the previous pressure and pressure increment. Applying them to the above matrix equation, the coefficient matrixes are deduced by the Galerkin method as follows:

$$
\left\{\begin{array}{l}
K_{i j}^{e}=\iint_{\Omega_{e}}\left\{\Phi_{\theta} H^{3} \frac{\partial N_{i}}{\partial \theta} \frac{\partial N_{j}}{\partial \theta}+\Phi_{z} H^{3}\left(\frac{R}{L}\right)^{2} \frac{\partial N_{i}}{\partial \bar{z}} \frac{\partial N_{j}}{\partial \bar{z}}+\xi N_{i}^{-} N_{j}^{-}\right\} \mathrm{d} \Omega_{e} \\
R_{i}^{e}=-\iint_{\Omega_{e}}\left\{\begin{array}{l}
\Phi_{\theta} H^{3} \frac{\partial P_{0}}{\partial \theta} \frac{\partial N_{i}}{\partial \theta}+\Phi_{z} H^{3}\left(\frac{R}{L}\right)^{2} \frac{\partial P_{0}}{\partial \bar{z}} \frac{\partial N_{i}}{\partial \bar{z}}+6 \Phi_{c}\left(U+U_{s}\right) N_{i} \frac{\partial H}{\partial \theta} \\
+N_{i} \frac{\sigma}{C} \frac{\partial\left[\Phi_{s}\left(U_{0}+U_{s}\right)\right]}{\partial \theta}+6 \Phi_{c} H N_{i}\left(\frac{\partial U_{s}}{\partial \theta}+\frac{R}{L} \frac{\partial W_{s}}{\partial \bar{z}}\right)+\xi N_{i}^{-} P_{0}^{-}
\end{array}\right\} \mathrm{d} \Omega_{e}
\end{array}\right.
$$

where $N_{i}$ and $N_{j}$ are the shape function of the four-node element with $i, j=1,2,3,4$. And the shape function can be expressed as

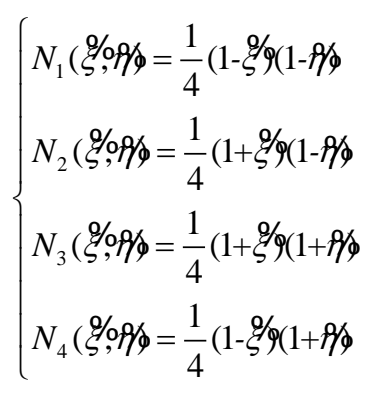

where $\mathcal{G}$ and $P /$ are the normalized coordinate of the element, $0 \leq \mathcal{F}, 9 / \propto<1$.

\subsection{Friction torque and friction coefficient}

The oil shear force is

$$
F_{f}=R \int_{0}^{L} \int_{0}^{2 \pi} \tau_{\theta z} \mathrm{~d} \theta \mathrm{dz}
$$

where $\tau_{\theta z}$ is the fluid shear stress, and $\tau_{\theta z}=\frac{\eta\left(u_{0}+u_{s}\right)}{h}\left(\phi_{f}+\phi_{f s}\right) \pm \phi_{f p} \frac{h}{2} \frac{\partial p}{R \partial \theta}$. \pm is used for the oil-shaft friction torque (+) and oil-bush friction torque (-). $\phi_{f} 、 \phi_{f s} 、 \phi_{f p}$ are the coefficients considering surface roughness, which can be determined in Ref [26].

The friction torque $T_{f}$ is

$$
T_{f}=R F_{f}=R \int_{0}^{L} \int_{0}^{2 \pi} \tau_{\theta z} R \mathrm{~d} \theta \mathrm{dz}
$$

The hydrodynamic bearing forces in $x$ and $y$ directions are

$$
\left\{\begin{array}{l}
F_{h x}=R \int_{0}^{2 \pi} \int_{0}^{L} p(\theta, z) \cos \theta \mathrm{dzd} \theta \\
F_{h y}=R \int_{0}^{2 \pi} \int_{0}^{L} p(\theta, z) \sin \theta \mathrm{d} z \mathrm{~d} \theta
\end{array}\right.
$$

The load carrying capacity is $F_{h}=\sqrt{F_{h x}^{2}+F_{h y}^{2}}$. The friction coefficient $f$ can be expressed by

$$
f=F_{f} / F_{h}
$$

The load carrying capacity gain is calculated by $F_{G}=\left(F_{h_{-} \text {slip }}-F_{h_{-} \text {nonslip }}\right) / F_{h_{-} \text {nonslip }} \times 100 \%$. The reduction in friction coefficient is calculated by $f_{D}=-\left(f_{\text {slip }}-f_{\text {nonslip }}\right) / f_{\text {nonslip }} \times 100 \%$. The decrease in friction torque is calculated by $T_{D}=-\left(T_{f_{-}}\right.$slip $\left.T_{f_{-} \text {noslip }}\right) / T_{f_{-} \text {noslip }} \times 100 \%$. The subscripts slip and nonslip mean the parameters calculated with and without the wall slip. 


\section{Result and Discussion}

\subsection{Verification of the model}

The present model is validated by the data in Ref. [12] as shown in Fig. 2. The dimensionless parameters are used. Eccentricity ratio is $\varepsilon=0.8$, length to diameter ratio is $L / D=1$, dimensionless critical shear stress is $\bar{\tau}_{c}=2$. In the present work, the slip dimensionless length is set to be 0.0345 . The dimensionless hydrodynamic pressure is noted as $P=p \psi^{2} / 2 \mu \omega$.

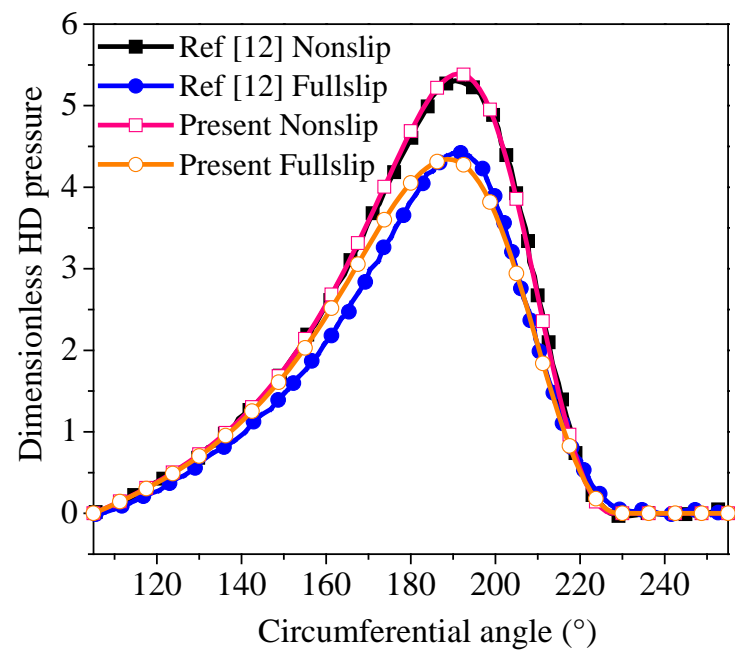

Fig. 2 Verification of the hydrodynamic pressure distribution in the middle plane

The effect of wall slip in axial direction in different slip zone shapes is shown in Fig. 3. The maximum slip velocity $W_{s}$ in axial direction occurs at both axial ends. With an increase in $\gamma$, the maximum $W_{s}$ increases. Meanwhile, the circumferential slip velocity $U_{s}$ occurs in a larger area. The negative effect of $W_{s}$ can be offset by the positive effect of $U_{s}$, and the effect of $W_{s}$ is still small. Therefore, the influence of slip velocity in axial direction is not considered in this analysis.

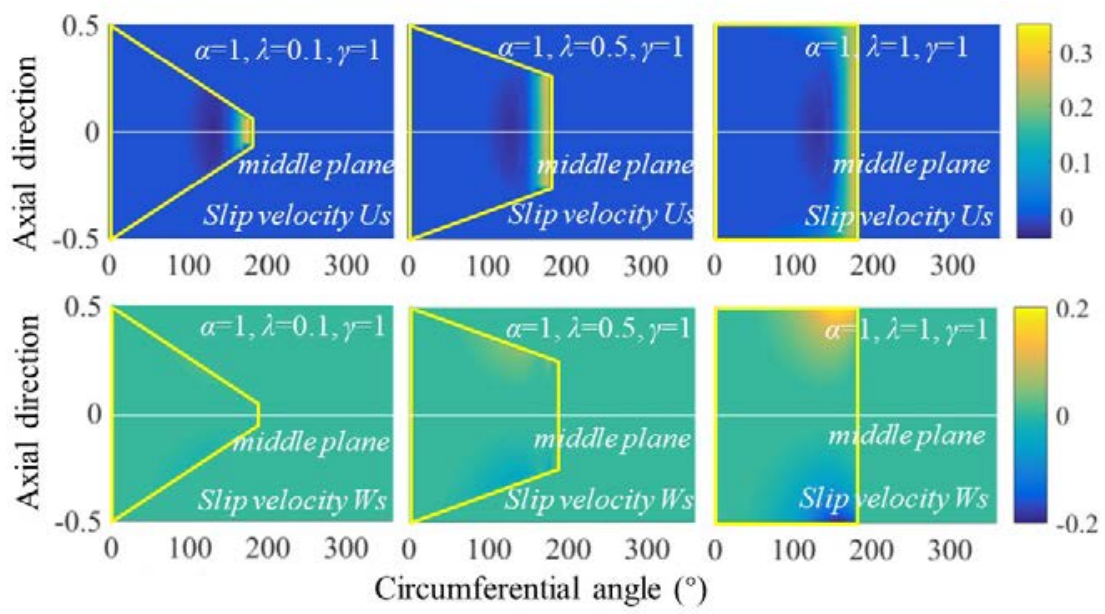

Fig. 3 The shape of the slip zone and the dimensionless slip velocity in (a) circumferential direction $U_{\mathrm{s}}$ and (b) axial direction $W_{s}$ at $\varepsilon=0.9$

\subsection{Optimization of slip zone in moderate eccentricity ratio}

Without any special notification, the common parameters used in this simulation are listed in Table 1. For different configurations of slip zone, the effect of slip length is not regular. Here we set the slip length to be $b=10 \mu \mathrm{m}$. The critical shear stress is $1126.94 \mathrm{~Pa}$ (for dimensionless $\bar{\tau}=0.28$ ) based on the oil-PTFE interface measured by Ref [3]. 
Table 1 Parameters of simulation

\begin{tabular}{|c|c|}
\hline Parameters & Values \\
\hline Shaft radius $R$, mm & 50 \\
\hline Bearing length $L$, mm & 80 \\
\hline Length to diameter ratio, $L / D$ & 0.8 \\
\hline Radial clearance $C, \mu \mathrm{m}$ & 145 \\
\hline Eccentricity ratio $\varepsilon$ & 0.5 \\
\hline Rotational speed $N$, rpm & 4000 \\
\hline Viscosity at $40^{\circ} \mathrm{C} \eta, \mathrm{Pa} \cdot \mathrm{s}$ & 0.0277 \\
\hline Dimensionless critical shear stress $\bar{\tau}$ & 0.28 \\
\hline Wall slip length $b, \mu \mathrm{m}$ & 10 \\
\hline Supply pressure $p_{\mathrm{s}}$, MPa & $P_{s}=0$ \\
\hline
\end{tabular}

\subsubsection{Effect of slip zone location (coefficient $\alpha$ )}

Fig. 4 shows the effect of coefficient $\alpha$ on the hydrodynamic behavior with the constant parameters of $\lambda=0.5\left(\beta=3.64^{\circ}\right)$ and $\gamma=0.8$. The pressure distribution is obviously affected by the slip zone coefficient $\alpha$ in both maximum value and pressure distribution (Fig. 4a). The dimensionless circumferential slip velocity $U_{s}$ in the middle plane variates with the slip zone coefficient $\alpha$ (Fig. 4b). For $\alpha>1, U_{s}$ increases first and then decreases in circumferential direction, reaching the maximum value at the position of minimum film thickness. According to Eq.(5), this is caused by the film thickness that changes inversely with $U_{s}$. Moreover, the hydrodynamic (HD) pressure is zero in the cavitation zone, but the slip velocity is not zero for $\alpha>1$, which is caused by shaft velocity. Since the shaft velocity is constant, the slip velocity in the cavitation zone is almost the same for different coefficient $\alpha$.

In Fig. 4 (c) and (d), the slip zones at $\alpha<1.0$ lead to a positive effect on hydrodynamic behavior by improving the load carrying capacity, decreasing the friction coefficient, and reducing slightly the friction torque in both oil-shaft and oil-bush interfaces. An optimal value of $\alpha=0.85$ is observed, which generates an increase of $3.4 \%$ in the load carrying capacity, a reduction of $4.1 \%$ in the friction coefficient. However, for $\alpha>1.0$, the slip zone generates a decrease in the load capacity and a rise in both the friction coefficient and friction torque. Therefore, it is better to set the slip zone at the convergent region of the film, instead of the divergent region (in Fig. 1).
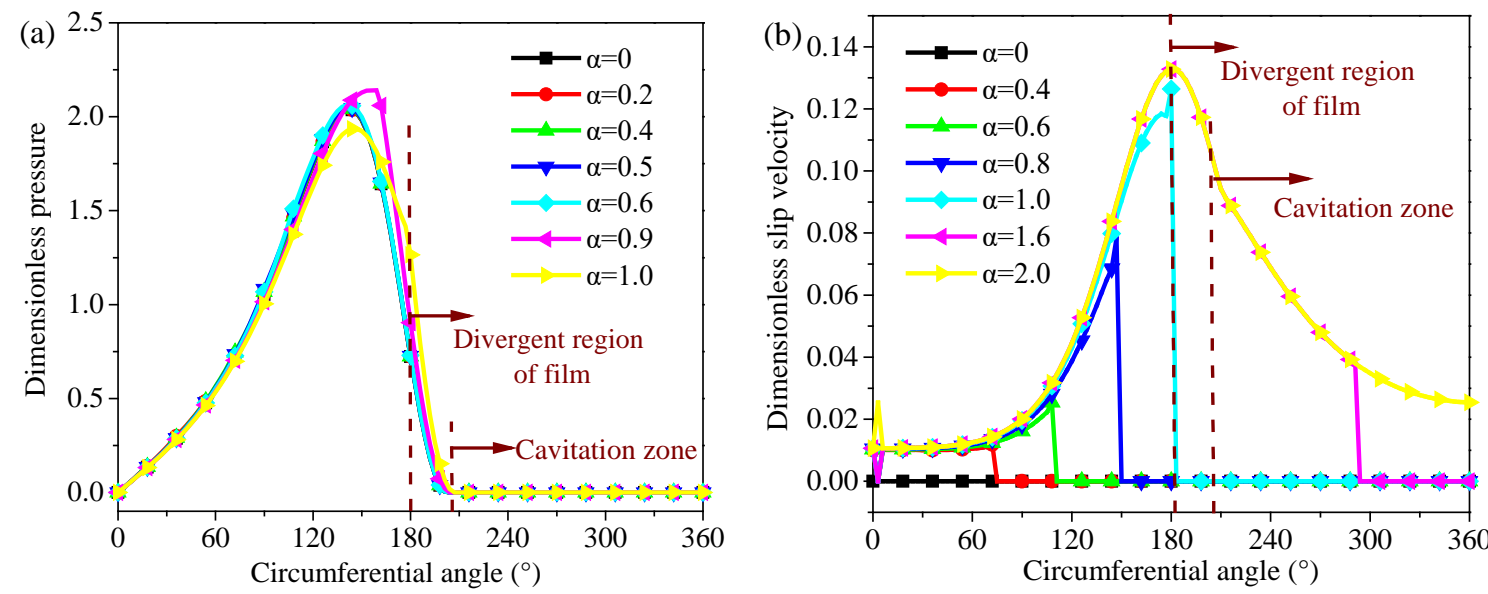

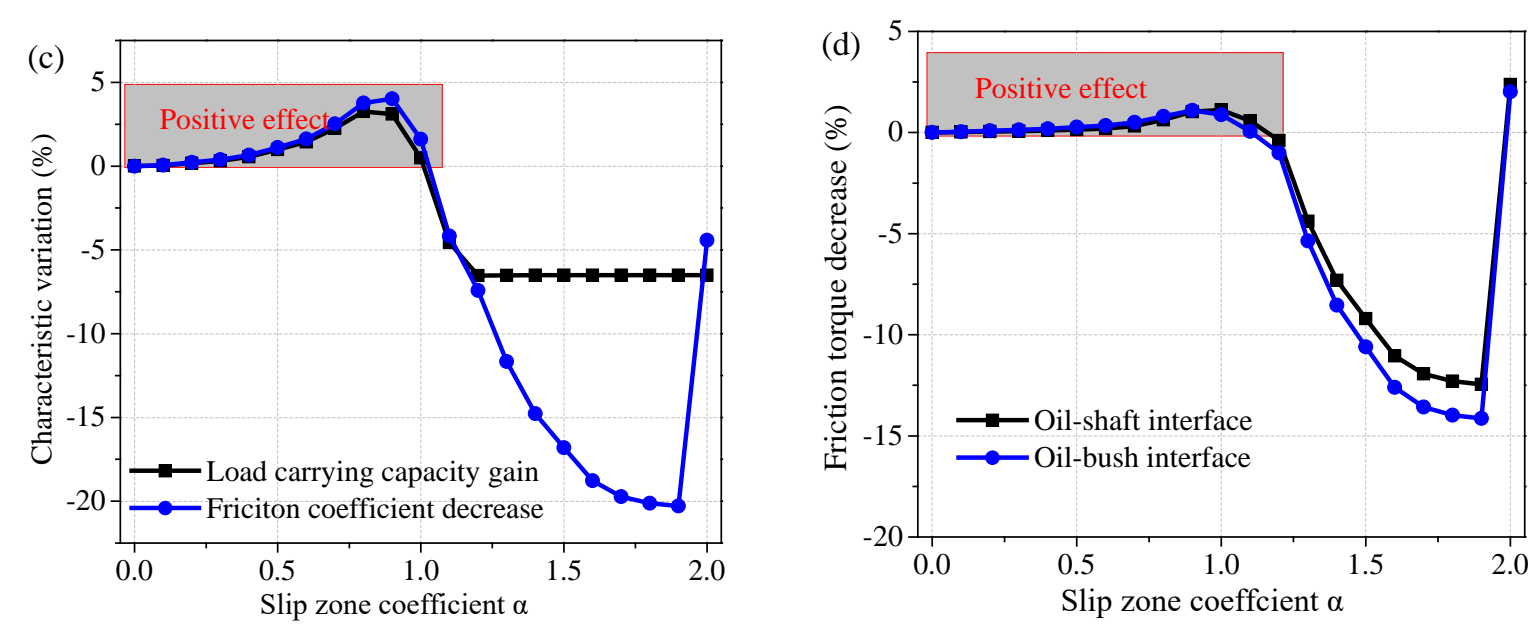

Fig. 4 The effect of slip zone coefficient $\alpha$ on the hydrodynamic (HD) behavior of journal bearings in moderate eccentricity ratio, (a) HD pressure $P$ in middle plane, (b) dimensionless slip velocity in circumferential direction $U_{s}$, (c) load carrying capacity gain $F_{G}$ and friction decrease $f_{D}$, and (d) friction torque decrease at oil-shaft $\left(T_{S D}\right)$ and oil-bush interfaces $\left(T_{b D}\right)$

\subsubsection{Effect of slip zone shape (coefficients $\lambda$ and $\gamma$ )}

Fig. 5 shows the effect of coefficient $\lambda$ on the hydrodynamic behavior with the constant parameters of $\gamma=0.8$ by setting the optimal slip zone coefficient $\alpha=0.85$ for $\varepsilon=0.5$. It demonstrates that the performance of journal bearings is better improved with large coefficient $\lambda$ (in Fig. 5a). For $\lambda=1.2$, the load carrying capacity gain climbs up to $4.8 \%$, meanwhile the friction coefficient drops by $5.9 \%$ with $1.5 \%$ reduction in friction torque. In addition, the coefficient $\lambda$ has little influence on the hydrodynamic (HD) pressure and slip velocity $U_{s}$ in the middle plane (Fig. $5 \mathrm{~b}$ and c), but it significantly affect the distribution of slip velocity $U_{s}$ (Fig. $5 \mathrm{~d}$ ), leading to the improvement of the bearings. Therefore, in order to better improve the performance of journal bearings, it is necessary to choose large coefficient $\lambda$ (or large $L_{1}$ ) to enlarge the action range of slip zone.
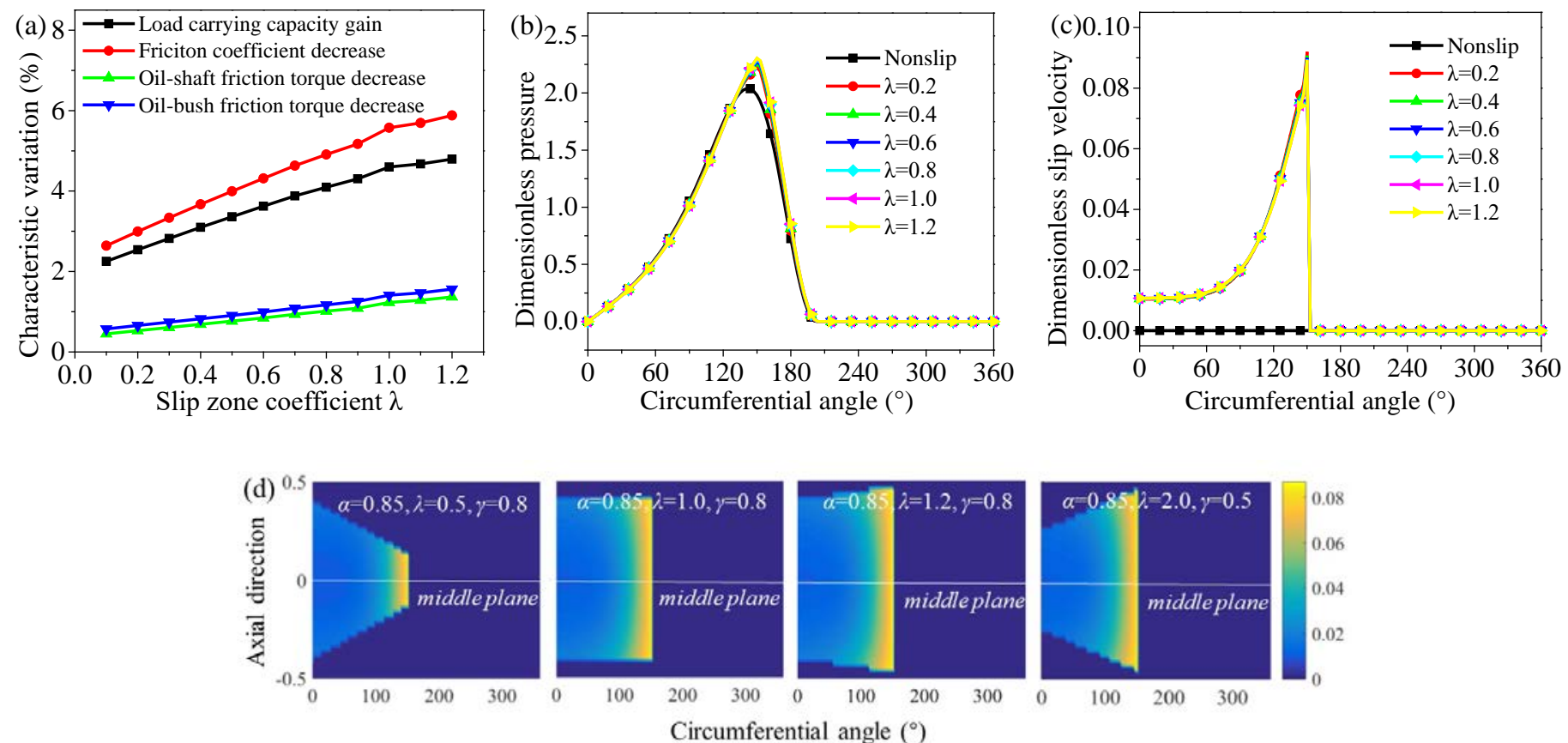

Fig. 5 Effect of slip zone coefficient $\lambda$ on the hydrodynamic (HD) behavior of journal bearings, (a) the load carrying capacity gain $F_{G}$, friction coefficient decrease $f_{D}$ and friction torque decrease $T_{D}$, (b) the HD pressure $P$ in middle plane, (c) the circumferential slip velocity $U_{s}$ in middle plane, (d) the distribution of slip velocity $U_{s}$ in different shapes of slip zone

Fig. 6 shows the effect of slip zone coefficient $\gamma$ on the hydrodynamic behavior of journal bearings with optimal slip zone 
coefficient of $\alpha=0.85$ for $\varepsilon=0.5$. $\lambda$ is set to be $1 / \gamma$, so the end width $L_{1}$ of the slip zone is fixed to be the widest value. The distribution of slip velocities $U_{\mathrm{s}}$ change with slip zone shapes based on the coefficient $\gamma$ (Fig. 6a). Fig. 6 (b) shows the increasing of $\gamma$ can slightly increase the load carrying capacity gain (with maximum $F_{G}=1.5 \%$ ) and the reduction of friction coefficient (with maximum $f_{D}=2 \%$ ), as well as the reduction of friction torque (less than $1 \%$ ). The coefficient $\gamma$ mainly affects the front part of the slip zone where slip velocity is much smaller than the end part, so its effect is not so significant. However, it is better to widen the slip zone in the inlet region, i.e. enlarge $L_{s 1}$, to augment the inlet flow rate.
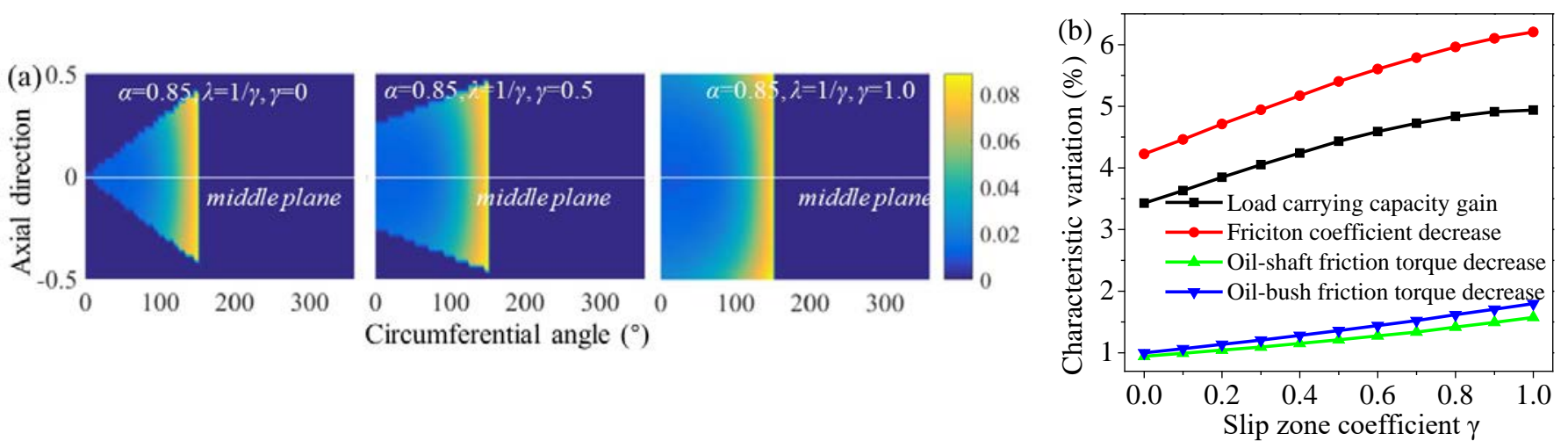

Fig. 6 The effect of slip zone coefficient $\gamma$ on the hydrodynamic behavior of journal bearings, (a) the distribution of slip velocity $U_{s}$ in different slip zone shapes, and (b) the load carrying capacity gain $F_{G}$, friction coefficient decrease $f_{D}$ and decrease of friction torque $T_{D}$

\subsubsection{Effect of eccentricity ratio $\varepsilon$}

Fig. 7 shows the effect of eccentricity ratio on the hydrodynamic behavior. The slip zone coefficients are chosen as $\alpha=0.85, \lambda=0.5$ and $\gamma=0.8$. Slip length is $b=10 \mu \mathrm{m}$. The partial slip zone behaviors much better in low eccentricity ratio than that in high eccentricity ratio (Fig. 7a). For $\varepsilon=0.1 \sim 0.8$, the partial slip zone significantly augment the load carrying capacity and reduced the friction coefficient with a slight change in friction torques. However, when $\varepsilon$ rises up to 0.9 , the performance of partial slip zone is negative by diminishing the load carrying capacity and increasing the friction coefficient. It can be seen in Fig. 7 (b), the slip velocity is large in low eccentricity ratio at the front part of slip zone. In high eccentricity of $\varepsilon=0.9$, negative slip velocity appears, i.e. the slip velocity goes into the opposite direction of the shaft speed, resulting in the decrease of the load carrying capacity. In order to improve the performance of the bearings, it is necessary to further optimize the slip zone coefficients in high eccentricity ratio.
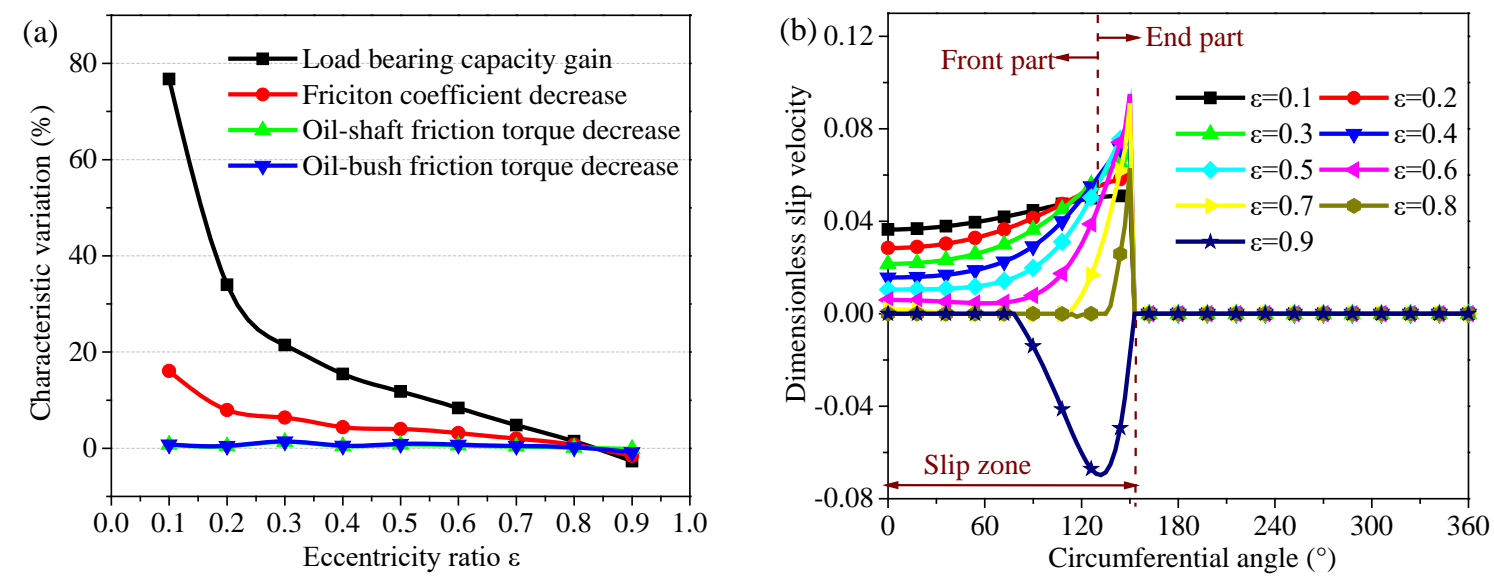

Fig. 7 Effect of eccentricity ratio on the hydrodynamic (HD) behavior, (a) the load-carrying load gain $F_{G}$, friction coefficient decrease $f_{D}$ and friction torque decrease $T_{D}$, and (b) the dimensionless circumferential slip velocity $U_{s}$ in the middle plane 


\subsection{Optimization of slip zone in high eccentricity ratio}

This part mainly focuses on the optimization of journal bearings in high eccentricity ratio. The common parameters used are eccentricity ratio $\varepsilon=0.9$, slip zone coefficients $\lambda=0.5$ and $\gamma=0.8$, slip length $b=10 \mu \mathrm{m}$, dimensionless critical shear stress $\bar{\tau}_{c}$ $=0.28$.

\subsubsection{Effect of slip zone location (coefficient $\alpha$ )}

Fig. 8 shows the effect of slip zone coefficient $\alpha$ on hydrodynamic behavior at the high eccentricity of $\varepsilon=0.9$. For $\alpha>1.0$, the load carrying capacity and friction coefficient are much worse than that of $\alpha<1.0$ (Fig. 8a), so it is better to set $\alpha<1.0$. In the positive region of $\alpha=0.91 \sim 1.0$, slip zone could improve the load carrying capacity with the maximum gain of $4.5 \%$, while the friction coefficient decrease $f_{D}$ rises up to the peak value of $2.4 \%$ during $\alpha=0.95 \sim 0.98$ (Fig. 8b). Fig. 8 (c) indicates that the oil-bush friction torque does not always decrease in the positive region, but the increment of friction torque is smaller than $2 \%$, so in high eccentricity ratio, the load carrying capacity and friction coefficient are used to obtain the optimal partial slip zone.
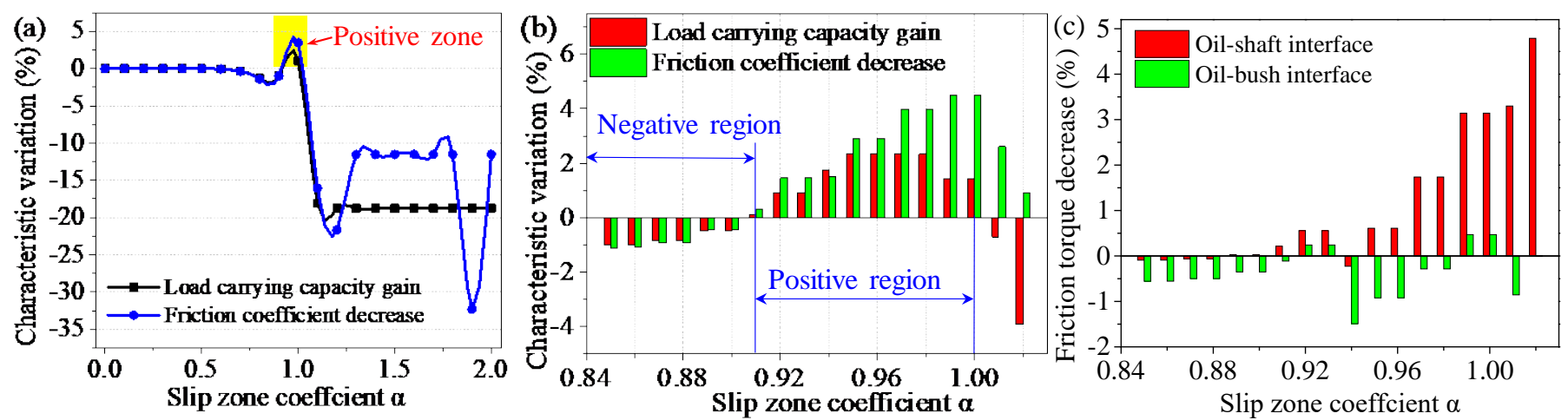

Fig. 8 Effect of slip zone coefficient $\alpha$ on the hydrodynamic behavior under high eccentricity ratio of $\varepsilon=0.9$, (a) the load carrying capacity gain $F_{G}$ and friction coefficient decrease $f_{D}$, (b) the load carrying capacity gain $F_{G}$ and friction coefficient decrease $f_{D}$ in positive region, and (c) friction torque $T_{D}$ decrease in positive region

\subsubsection{Effect of slip zone shape (coefficient $\lambda$ and $\gamma$ )}

Fig. 9 shows the effect of coefficient $\lambda$ on the performance of journal bearings with the eccentricity ratio of $\varepsilon=0.9$, the slip zone coefficient of $\alpha=1.0$. It can be seen that the load carrying capacity gain $F_{G}$ and friction coefficient reduction $f_{D}$ increase with coefficient $\lambda$ (Fig. 9a). $F_{G}$ and $f_{D}$ reach their maximum values of $4.8 \%$ and $11 \%$ respectively at $\lambda=1.2$. This means that the slip zone with a wider length $L_{1}$ in axial direction works better. For constant $\alpha$, large coefficient $\lambda$ causes a little increase in maximum hydrodynamic pressure (Fig. 9b). In Fig. 9 (c), before $\theta=150^{\circ}$ slip velocity is negative, while it becomes positive after $\theta=150^{\circ}$.
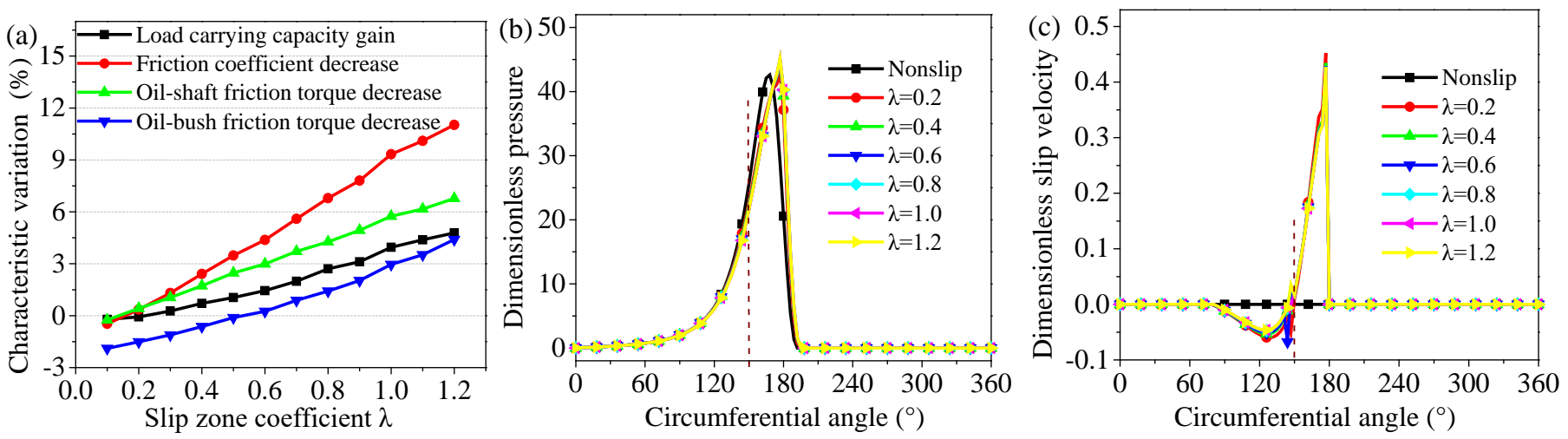

Fig. 9 Effect of slip zone coefficient $\lambda$ on the hydrodynamic (HD) behavior of journal bearings, (a) the load carrying capacity gain $F_{G}$, friction coefficient decrease $f_{D}$ and friction torque decrease $T_{D}$, (b) the HD pressure $P$ in middle plane, and (c) the 
dimensionless circumferential slip velocity $U_{s}$ in middle plane

Fig. 10 shows the effect of slip zone coefficient $\gamma$ on the hydrodynamic behavior of journal bearings with coefficients of $\alpha=1$ and $\lambda=1 / \gamma$. For more obvious effect, larger eccentricity ratio of $\varepsilon=0.92$ is used. It is clear that the increasing coefficient $\gamma$ can improve the load carrying capacity slightly with $F_{G}<1 \%$, and lower friction coefficient and friction torque with $f_{D}<1.23 \%$ and $T_{D}<1.4 \%$ respectively. Moreover, coefficient $\gamma$ has little influence on hydrodynamic pressure and slip velocity $U_{s}$ in the middle plane (Fig. 10b and c). This is because the shape change caused by coefficient $\gamma$ mainly influences the slip zone where the slip velocity is close to zero and make little change in the pressure distribution, and the variation of $\gamma$ could not offset the negative zone of slip velocity $U_{s}$ (Fig. 10d). Despite the subtle influence of $\gamma$, it is still beneficial to widen the front size of the slip zone, i.e. enlarge $L_{s 1}$.
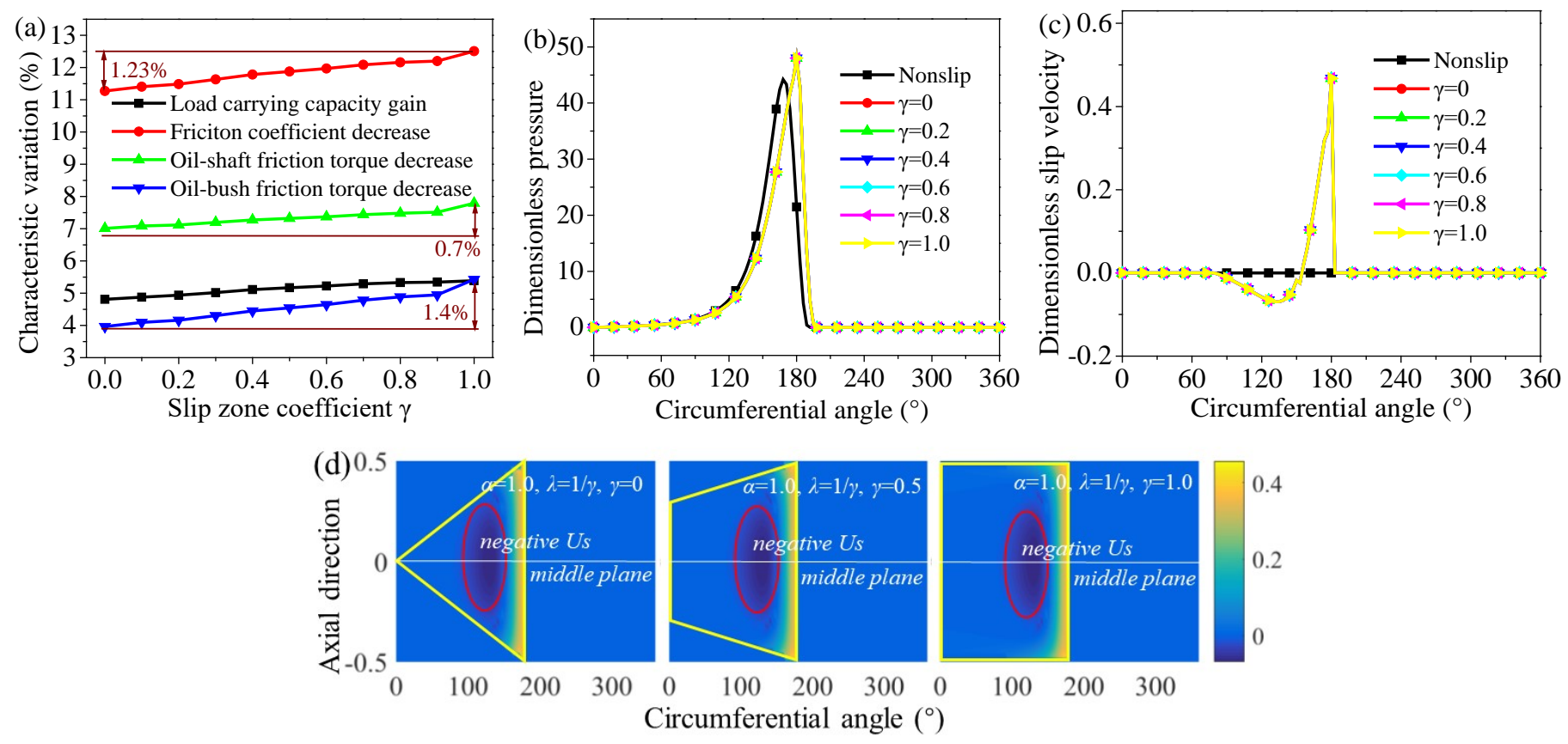

Fig. 10 Effect of slip zone coefficient $\gamma$ on the hydrodynamic behavior of journal bearings in high eccentricity ratio, (a) the load carrying capacity gain $F_{G}$, friction coefficient decrease $f_{D}$ and friction torque decrease $T_{D}$, (b) the HD pressure $P$ in middle plane, (c) the slip velocity $U_{s}$ in middle plane, (d) the distribution of slip velocity $U_{s}$

The influence of slip zone shape, i.e. the coefficients of $\lambda$ and $\gamma$ at high eccentricity ratio is similar to that in moderate eccentricity ratio. It is helpful to increase both the coefficients of $\lambda$ and $\gamma$. That is to say setting $\lambda=1$ and $\gamma=1$ and the optimal shape of the slip zone is rectangular.

\subsubsection{Effect of high eccentricity ratio $\varepsilon$}

Fig. 11 shows the effect of high eccentricity ratio on the hydrodynamic behavior with the optimized slip zone parameters of $\alpha=1, \lambda=1$, and $\gamma=1$, slip length $b=5 \mu \mathrm{m}$. As shown in Fig. 11(a), the load carrying capacity gain $F_{G}$ increases with the rise of $\varepsilon$. The friction coefficient decrease $f_{D}$ experiences rise and meets the largest value of $12.5 \%$ at $\varepsilon=0.96$. However, there is a special point of $\varepsilon=0.93$, whose performance is better than the two adjacent points of $\varepsilon=0.92$ and $\varepsilon=0.94$. This is because the present slip zone parameters are not optimal for all the eccentricity ratios. In this case, these parameters can best improve the performance of $\varepsilon=0.93$ and it is marked as a special point. For $\varepsilon>0.96$, the $F_{G}$ and $f_{D}$ go lower, but it can be concluded that the rectangular slip zone can still improve the performance of journal bearings for $\varepsilon=0.9 \sim 0.97$. As shown in Fig. 11 (b) and (c) and Eq.(5), in slip zone 1 the pressure gradient $\mathrm{d} p / \mathrm{d} \theta$ is close to zero and the film thickness is high, so the slip velocity is almost zero. In slip zone 2, though film thickness becomes smaller, the slip velocity is still negative because of the increasing $\mathrm{d} p / \mathrm{d} \theta$. In slip zone $3, \mathrm{~d} p / \mathrm{d} \theta$ is quite large, but the film thickness also becomes small enough to offset the negative effect of $\mathrm{d} p / \mathrm{d} \theta$, so there is a sharp increase in slip velocity. 

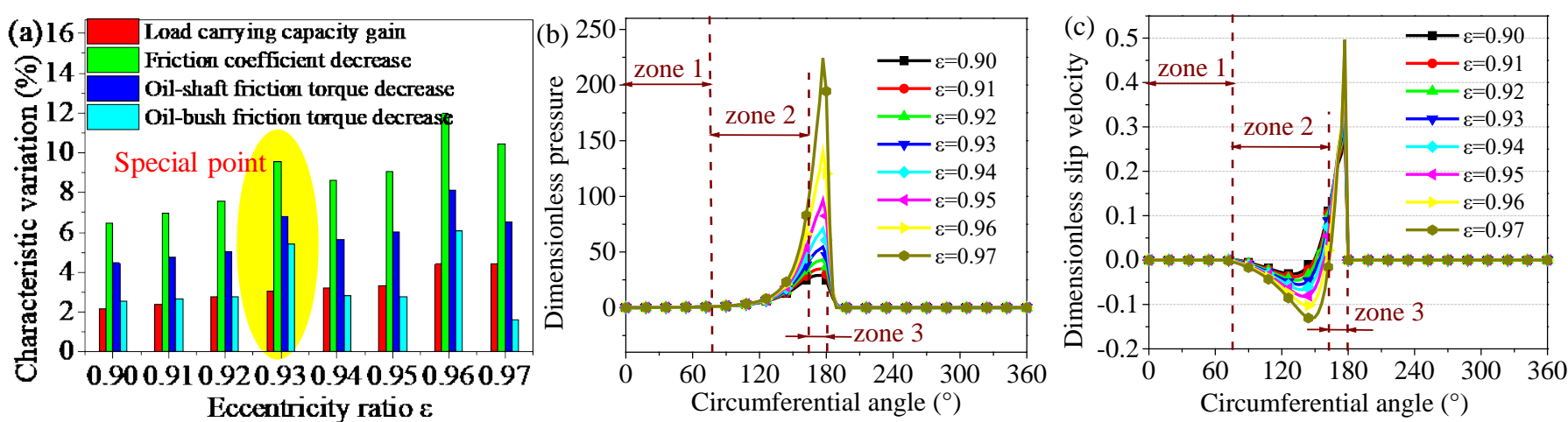

Fig. 11 Effect of high eccentricity ratio on hydrodynamic behavior of journal bearings, (a) effect on load carrying capacity gain $F_{G}$, friction coefficient decrease $f_{D}$ and friction torque decrease $T_{D}$, (b) effect on hydrodynamic (HD) pressure in the middle plane, and (c) effect on circumferential dimensionless slip velocity $U_{s}$ in the middle plane

\subsection{Considering operating conditions}

In this part, the optimized slip zone coefficients of $\lambda=1$ and $\gamma=1$ are selected. The influence of attitude angle in the high eccentricity ratio is considered. The fixed coordinate system of $\Theta$ is applied (Fig. 1b), and its origin of $\Theta=0$ is the vertical direction of $Y=0$. The slip zone starts from the system origin of $\Theta=0$, instead of $\theta=0$. In the fixed coordinate system $\Theta$, attitude angle $\varphi$ is not zero. As the partial slip zone of $\Theta_{\text {slip }}=0 \sim \varphi$ locates at the end of the divergent region of the film, its influence can be neglected. Therefore, in high eccentricity ratio the optimal $\alpha$ still located at $0.9 \sim 1.0$. The slip zone is better to end at the position of $\Theta_{\text {end }}=\pi+\kappa \varphi(\kappa \in[0,1])$, i.e. $B_{\mathrm{s}}=\pi+\kappa \varphi, \alpha=\left(B_{\mathrm{s}}-\varphi\right) / B_{1}$.

The optimized parameters of $\varepsilon=0.94$ are selected for further exploring the performance of journal bearing with eccentricity ratios ranging from 0.4 to 0.97 as shown in Fig. 12. The slip length is $b=5 \mu \mathrm{m}$. The slip zones endings are set to be $\Theta_{\text {end }}=\pi, \Theta_{\text {end }}=\pi+0.25 \varphi, \Theta_{\text {end }}=\pi+0.5 \varphi, \Theta_{\text {end }}=\pi+0.75 \varphi$, and $\Theta_{\text {end }}=\pi+1.0 \varphi$, corresponding to $\kappa=0, \kappa=0.25, \kappa=0.50, \kappa=0.75$ and $\kappa=1.00$ respectively. The nonslip attitude angle of $\varepsilon=0.94$ is $\varphi=20.3^{\circ}$, so the corresponding values of $\alpha$ are $0.889,0.915,0.943$, 0.971 and 0.999 . For $\varepsilon<0.94$, the selected slip zone can raise the load carrying capacity and lower the friction coefficient (Fig. 12a and b). For $\kappa=0.75$ and $\kappa=1.00$, the partial slip zone better improves the bearing performance in different eccentricity ratios with the maximum load gain of $4.5 \%$ and the maximum friction coefficient decrease of $10 \%$. However, for $\varepsilon>0.94$, except $\kappa=0.5$, partial slip zone diminish the load carrying capacity in any case.

Usually with the increase in eccentricity ratio, the attitude angle drops, and the slip zone coefficient $\alpha$ rises. In Fig. 12(c), it can be seen for $\varepsilon<0.94$ and $\kappa=0.5 \sim 1$ the attitude angle goes down, so the exact value of coefficient $\alpha$ becomes higher. If the journal bearing always works in a constant heavy load, in order to get the optimal performance it is better to set the partial slip zone with the coefficient $\kappa$ ranging from 0.5 to 0.9 .
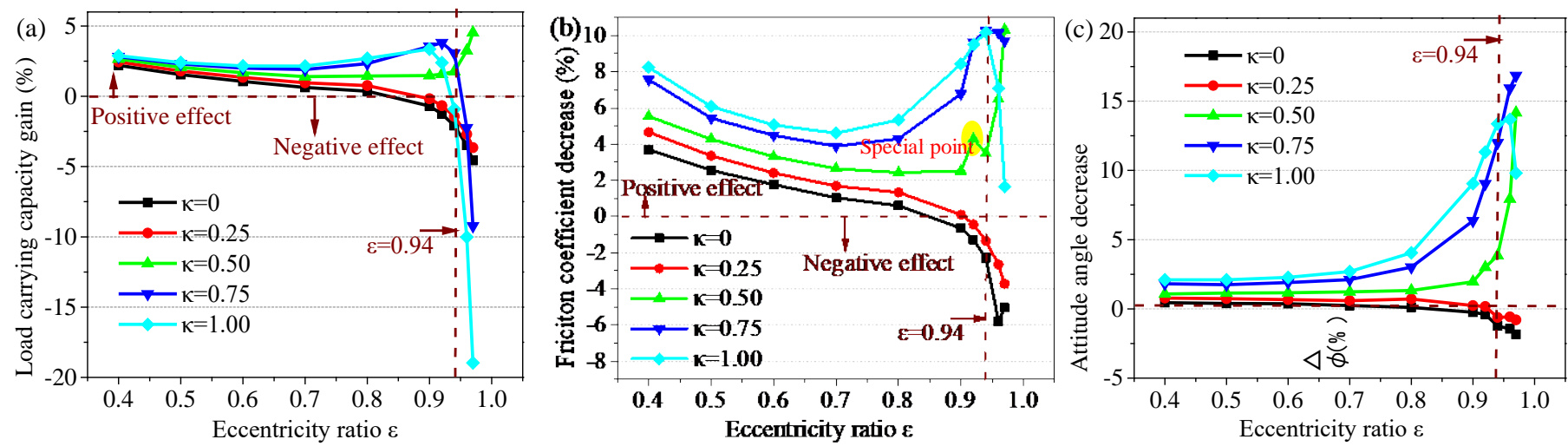

Fig. 12 The performance of journal bearing in partial slip zone in different eccentricity ratio, (a) the load carrying capacity gain $F_{G}$, (b) the friction coefficient decrease $f_{D}$, (c) the attitude angle, and (d) the decrease percentage of attitude angle 


\section{Conclusions}

In the present work, the improved performance of journal bearings with partial slip zone is obtained by optimizing slip zone shape at both moderate and high eccentricity ratios. The key conclusions are as follows:

(1) It is better to enlarge the wall slip zone in axial direction in the oil-wedge convergent zone, i.e. setting the slip zone in rectangular. For high eccentricity ratio, in circumferential direction, the optimal slip zone is located at the convergent region of film and the optimal coefficient $\alpha$ is approximately $0.9 \sim 1.0$ which is limited much more strictly than that in moderate eccentricity ratio.

(2) For the working condition of heavy load, it is better to locate partial slip zone during $\Theta=0 \sim \pi+\kappa \varphi$ with the coefficient of $\kappa=0.5 \sim 0.9$ in circumferential direction, where $\varphi$ is the attitude angle of the highest eccentricity ratio.

(3) In high eccentricity ratio, there exists a part of slip zone where the slip velocity goes in the opposite direction of the shaft speed, which weakens the performance of journal bearings.

As the influence of wall slip on journal bearings are very complex, the present model makes some hypotheses for simplification. Therefore, further studies would be needed considering the thermal and elastic deformation. Also, experiments need to be conducted for the application of the optimal slip zone.

\section{Acknowledgment}

This research is supported by the National Natural Science Foundation of China (No.51675120 and No.U1537214) and SelfPlanned Task (SKLRS201814B). The first author also wants to thank China Scholarship Council (No.201806120132) for the financial support of studying aboard.

\section{References}

[1] Senatore A, Rao TV. Partial Slip Texture Slider and Journal Bearing Lubricated With Newtonian Fluids: A Review. ASME J. Tribol 2018;140(4): 040801.

[2] Yu X, Meng Y, Tian Y, et al. Measurement of lubricant viscosity and detection of boundary slip at high shear rates. Tribol Int 2016; 94:20-5.

[3] Jin J. Theoretical and experimental research of Elasto-Metal-Plastic(EMP) journal bearings. Doctoral thesis, Shanghai university, China, 2005.

[4] Choo JH, Glovnea RP, Forrest AK, Spikes HA. A low friction bearing based on liquid slip at the wall. ASME J. Tribol 2007; 129(3):611-20.

[5] Kalin M, Velkavrh I, Vižintin J. The Stribeck curve and lubrication design for non-fully wetted surfaces. Wear 2009; 267(58):1232-1240.

[6] Ryan D, Evans J, David C, et al. Traction of Lubricated Rolling Contacts between Thin-Film Coatings and Steel. Tribol Trans 2008; 52(1):106-113.

[7] Rao TV, Rani AM, et l. Analysis of slider and journal bearing using partially textured slip surface. Tribol Int 2012; 56:121-8.

[8] Bailey NY, Cliffe KA, et al. Dynamics of a high speed coned thrust bearing with a Navier slip boundary condition. J. Eng Math 2016; 97(1):1-24.

[9] Tauviqirrahman M, Ismail R, Jamari J, Schipper DJ. Combined effect of texturing and boundary slippage in lubricated sliding contacts. Tribol Int 2013; 66:274-81.

[10] Fatu A, Maspeyrot P, Hajjam M. Wall slip effects in (elasto) hydrodynamic journal bearings. Tribol Int 2011; 44(7-8):868-877.

[11] Ma GJ, Wu CW, Zhou P. Influence of wall slip on the dynamic properties of a rotor-bearing system. Tribol Trans 2008; 51(2):204-12.

[12] Lv F, Rao Z, Ta N, Jiao C. Mixed-lubrication analysis of thin polymer film overplayed metallic marine stern bearing considering wall slip and journal misalignment. Tribol Int 2017; 109:390-7.

[13] Spikes H, Granick S. Equation for slip of simple liquids at smooth solid surfaces. Langmuir 2003; 19(12):5065-71. 
[14] Ismail S, Sarangi M. Effects of texture shape and fluid-solid interfacial slip on the hydrodynamic lubrication performance of parallel sliding contacts. P I Mech Eng J-J Eng 2014; 228(4):382-96.

[15] Zhang H, Hua M, Dong GN, Zhang DY, Chin KS. Boundary slip surface design for high speed water lubricated journal bearings. Tribol Int 2014;79:32-41.

[16] Cheng F, Ji W. A velocity-slip model for analysis of the fluid film in the cavitation region of a journal bearing. Tribol Int 2016; 97:163-172.

[17] Jao HC, Chang KM, Chu LM, et al. A modified average Reynolds equation for rough bearings with anisotropic slip. ASME J. Tribol 2016; 138(1): 011702.

[18] Ma GJ, Wu CW, Zhou P. Wall slip and hydrodynamics of two-dimensional journal bearing. Tribol Int 2007; 40(7):1056-66.

[19] Rao TV. Analysis of single-grooved slider and journal bearing with partial slip surface. ASME J. Tribol 2010; $132(1): 014501$.

[20] Fortier AE, Salant RF. Numerical analysis of a journal bearing with a heterogeneous slip/no-slip surface. ASME J. Tribol 2005; 127(4):820-5.

[21] Wang LL, Lu CH, et al. Study on the influence of critical shear stress on wall slip of spiral oil wedge journal bearing. P I Mech Eng J-J Eng 2012; 226(5):362-376.

[22] Wang LL, Lu CH, et al. The numerical analysis of the radial sleeve bearing with combined surface slip. Tribol Int 2012; 47:1004.

[23] Wu C, Zheng L. An average Reynolds equation for partial film lubrication with a contact factor. ASME J. tribol 1989; 111(1): 188-191.

[24] Habchi W, Eyheramendy D. Vergne P, et al. Stabilized fully-coupled finite elements for elastohydrodynamic lubrication problems. Adv Eng Softw 2012; 46(1):4-18.

[25] Cui S, Gu L, Wang L, et al. Numerical analysis on the dynamic contact behavior of hydrodynamic journal bearings during startup. Tribol Int. 2018;121:260-8.

[26] Patir N, Cheng HS. Application of average flow model to lubrication between rough sliding surfaces. J. Lubrication Tech 1979; 101(2): 220-229. 